\title{
تخطيط جداول الانتاج الرئيسية بأ ستخدام أسلوب برهجة الاعداد الصحيحة ذات القرارات الثنائية
}

\author{
م. مد عبد الجبار خضر بخيت \\ جامعة بغداد- كلية الادارة والاقتصاد الإن \\ قسم الاحصاء الآد والاء
}

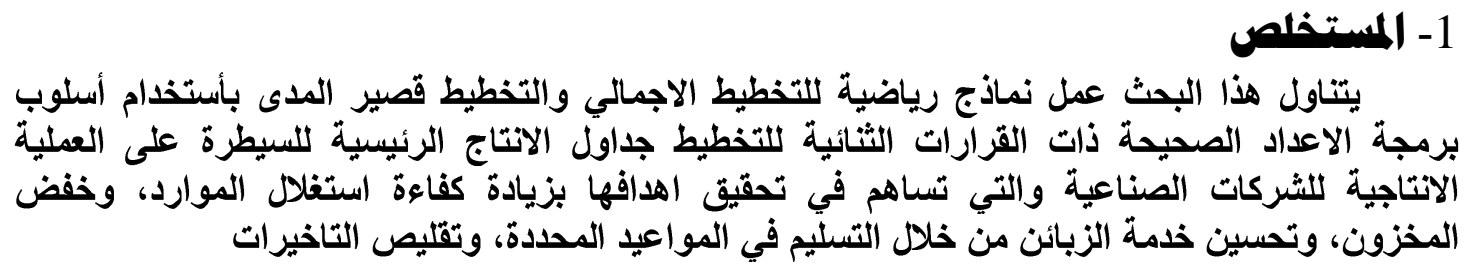

Abstract

This research include building mathematical models for aggregating planning and shorting planning by using integer programming technique for planning master production scheduling in order to control on the operating production for manufacturing companies to achieve their objectives of increasing the efficiency of utilizing resources and reduce storage and improving customers service through deliver in the actual dates and reducing delays. 


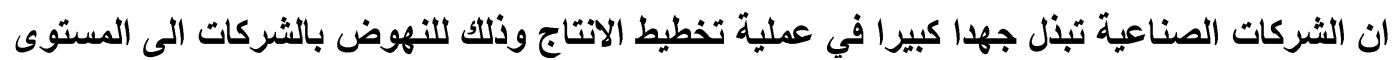

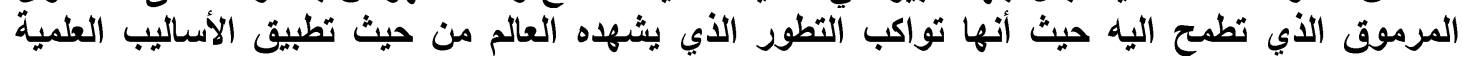

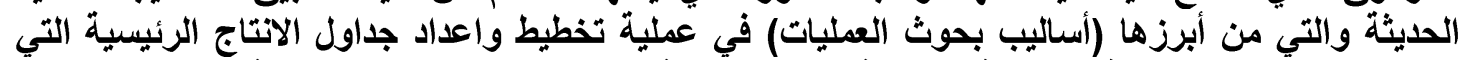

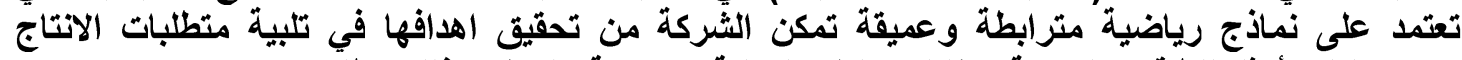

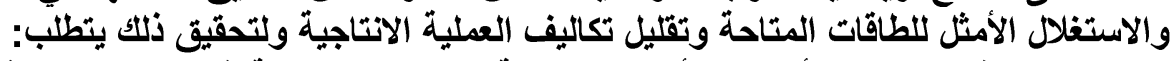

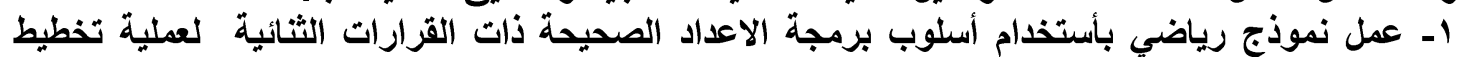

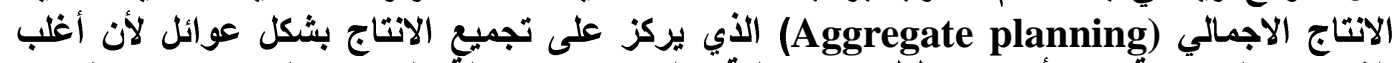

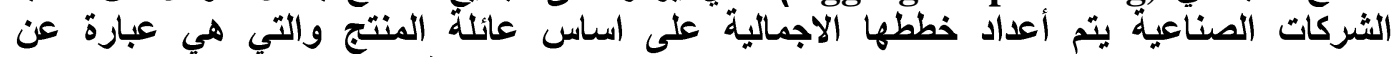

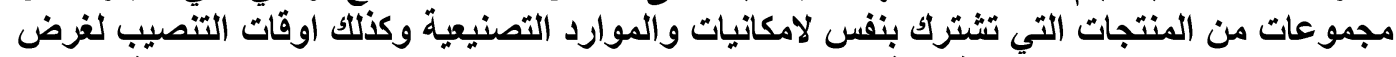

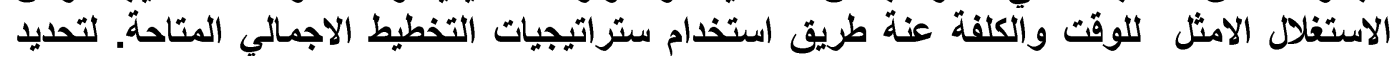
المتطلبات والامكانات المتاحة بشكل بأنكل اجمالي.

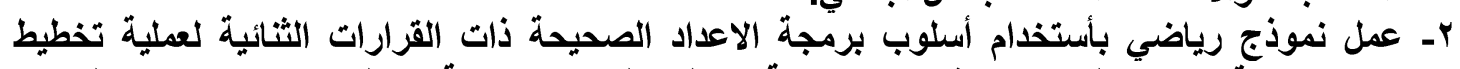

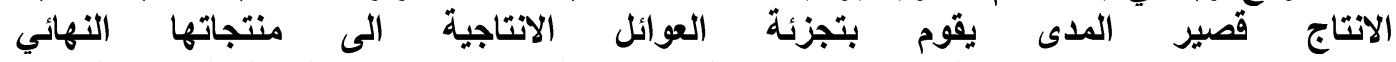

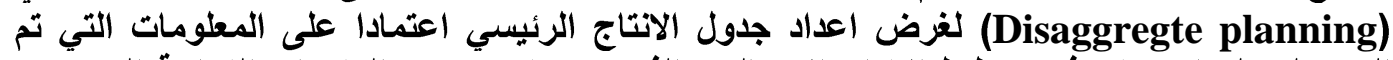

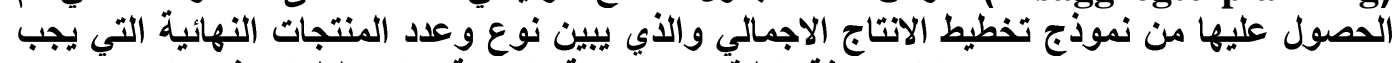

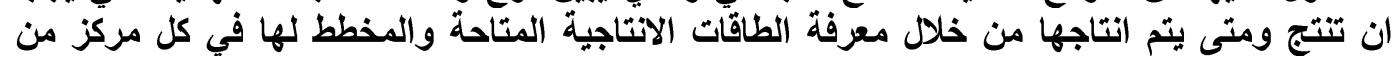
مراكز الانتاج.

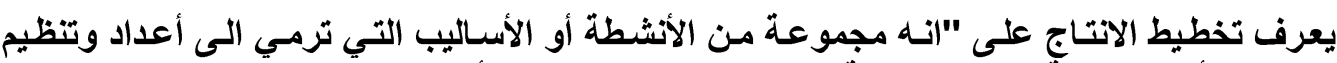

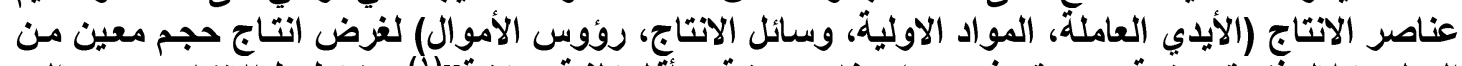

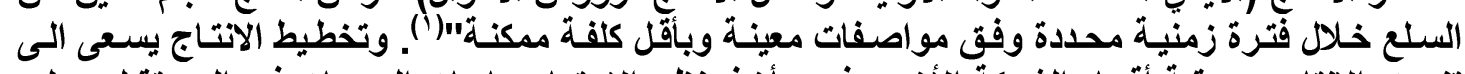

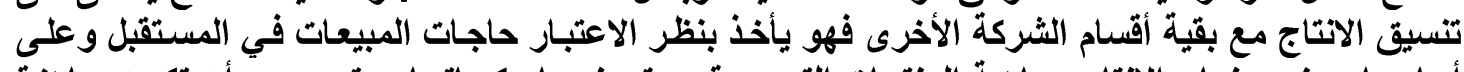

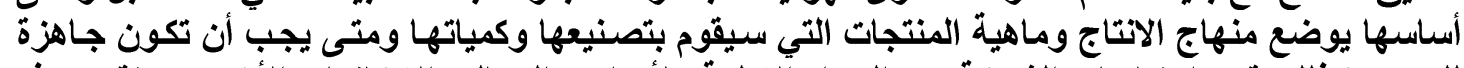

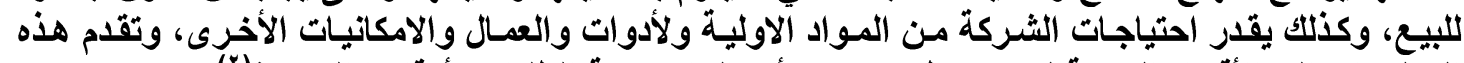

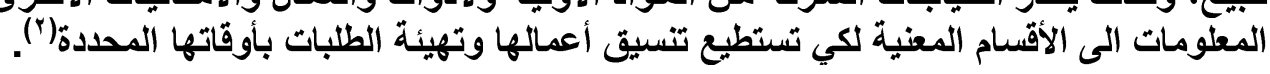

\section{r- برهجة الاعداد الصحيحة (Integer Programming)}

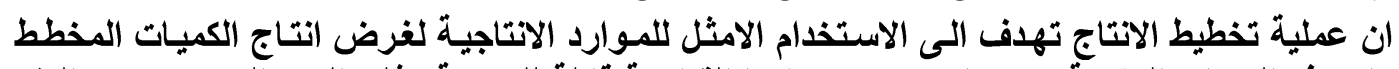

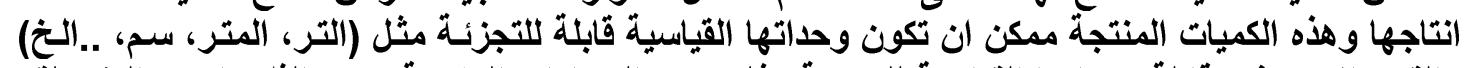

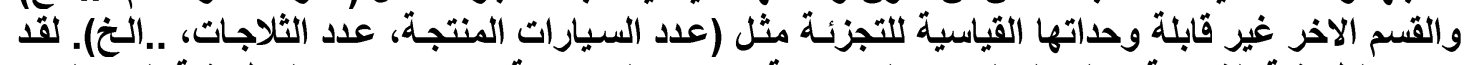

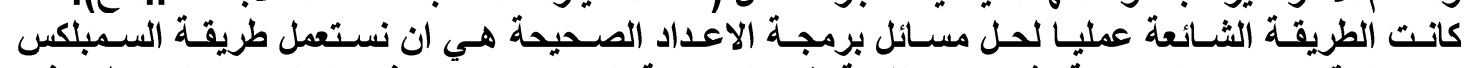

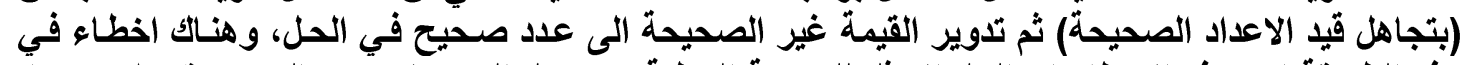

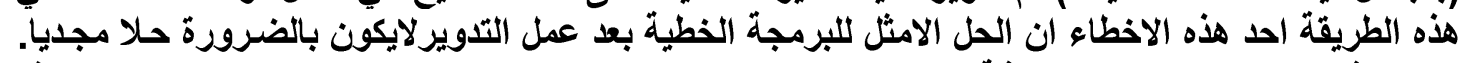

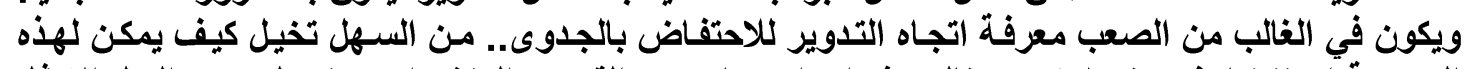

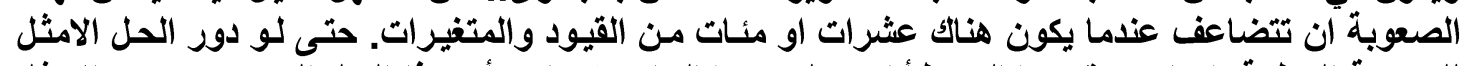

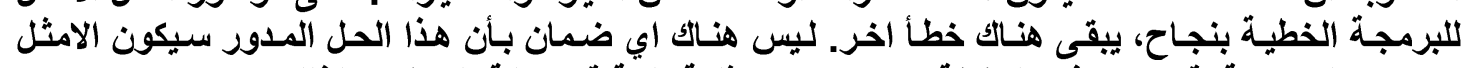

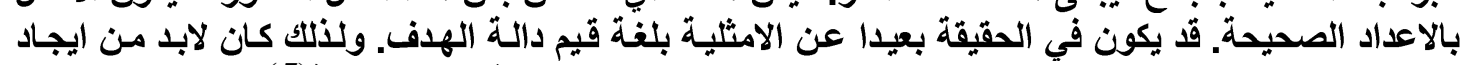

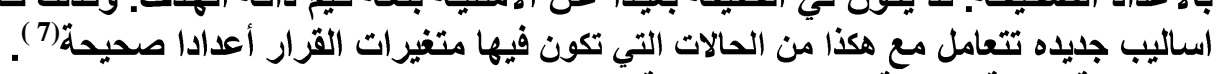

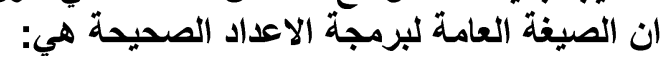




$$
\begin{aligned}
& \text { MAXorMinZ }=C_{1} X_{1}+C_{2} X_{2}+\ldots \ldots+C_{n} X_{n} \\
& a_{11} X_{1}+a_{12} X_{2}+\ldots \ldots+a_{1 n} X_{n}(\leq,=, \text { or } \geq) \cdot b_{1} \ldots \ldots \ldots \ldots \ldots .(3-2) \\
& a_{21} X_{1}+a_{22} X_{2}+\ldots \ldots \ldots \ldots+a_{2 n} X_{n} \ldots \ldots \ldots \ldots \ldots \ldots \ldots \ldots \ldots . .(3-3) \\
& a_{m 1} X_{1}+a_{m 2} X_{2}+\ldots .+a_{m n} X_{n}(\leq,=, \text { or } \geq) b_{m} \ldots \ldots \ldots \ldots \ldots . .(3-4) \\
& X_{1}, X_{2}, \ldots \ldots . X_{n} \geq 0 \text { and int eger }
\end{aligned}
$$

\section{צ- حالات برهجة الاعداد الصحيحة: هناك ثلاثة حالات لبرهجة الاعداد الصحيحة (8 ) :}

1- اذا كاتت الوحدات القياسية لجميع متغيرات القرارغير قابلة للتجزئة صحيحة فقي هذه الحالة الحاتة تسمي (Pure Integer Programming Problem)

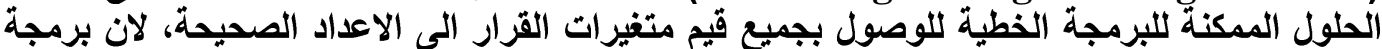

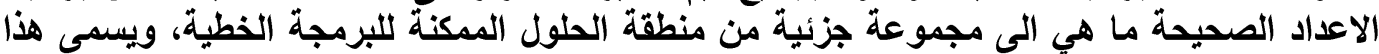

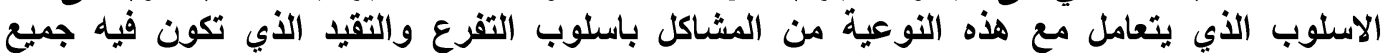
متغيرات القرار اعدادا صحيحة.

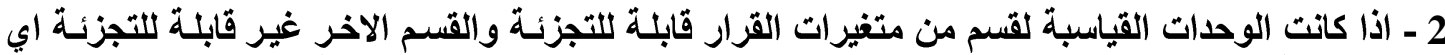

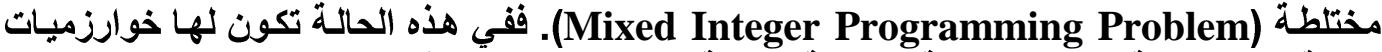
خاصة لقطع منطقة الحلول الممكنة للبرمجة الخطية للوصول بقسم من قيم متغيرات القرار الى الاعداد الصحيحة.

r- برمجة الاعداد الصحيحة للقرارات الثنائية.(I.P for binary decision).

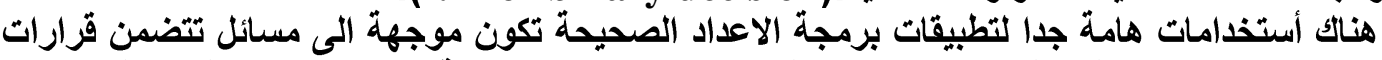

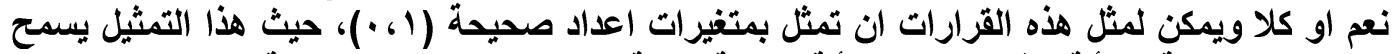

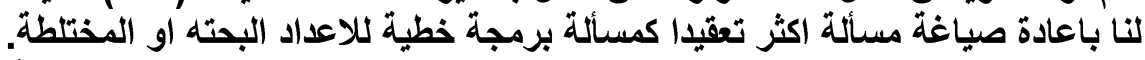

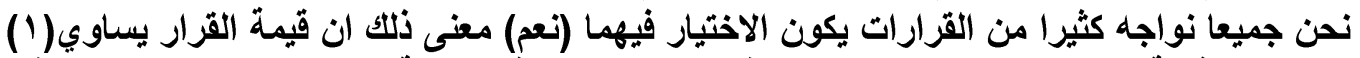

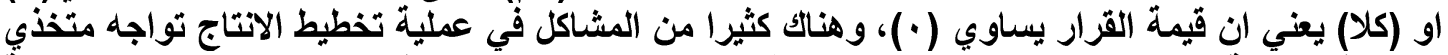

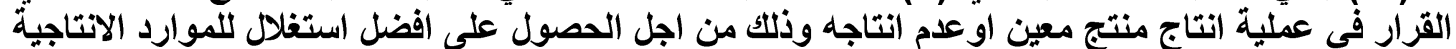

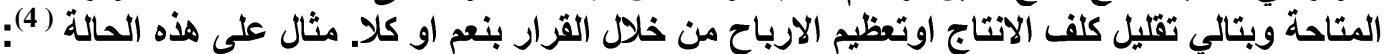

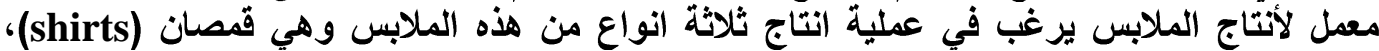

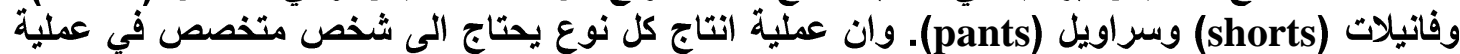

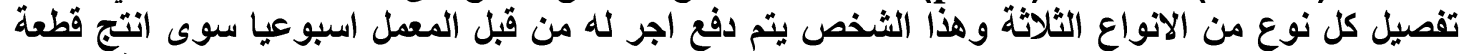

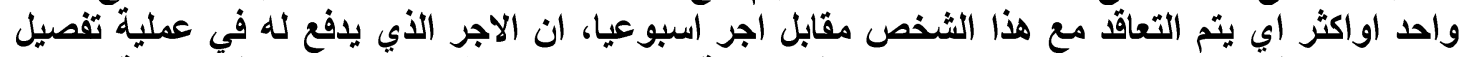

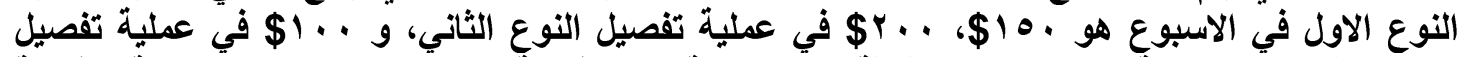

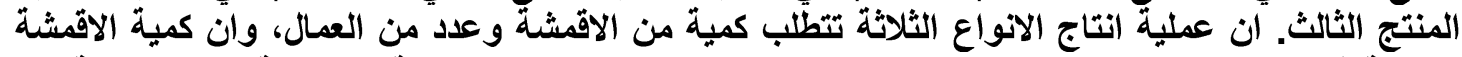

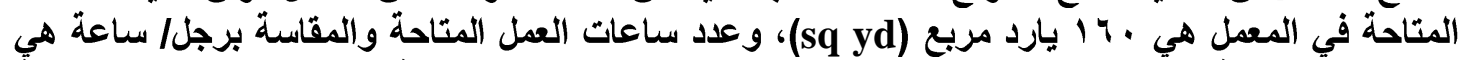

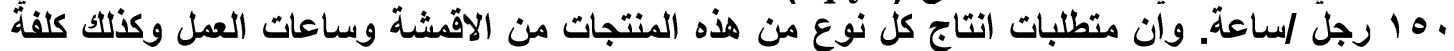

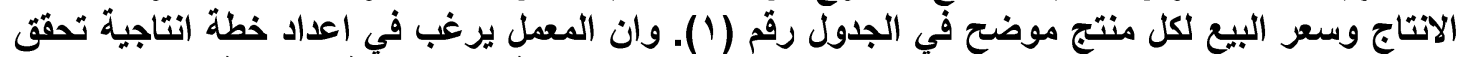

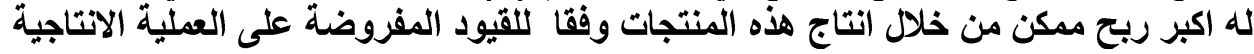


Table (1)

\begin{tabular}{|c|c|c|c|c|}
\hline Products-type & Labor (hours) & $\begin{array}{c}\text { Cloth } \\
\text { (squares yards) }\end{array}$ & Sales price & Variable cost \\
\hline Shirts & 3 & 4 & $\$ 12$ & $\$ 6$ \\
\hline shorts & 2 & 3 & $\$ 8$ & $\$ 4$ \\
\hline pants & 6 & 4 & $\$ 15$ & $\$ 6$ \\
\hline
\end{tabular}

لغرض صياغة المشكلة لابد من تعريف متغيرات القرار:

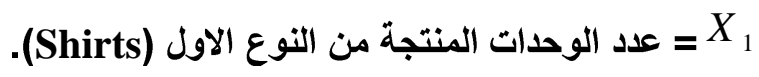

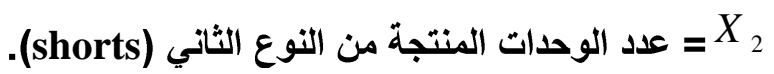

. $X_{3}$

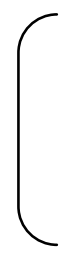

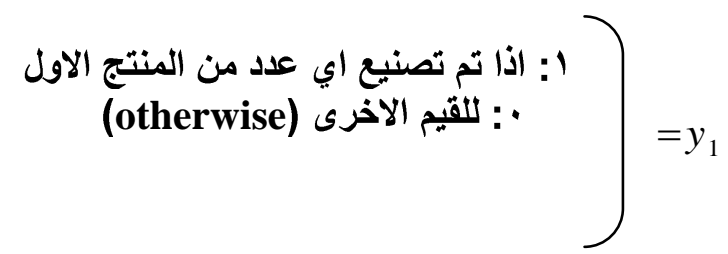

(

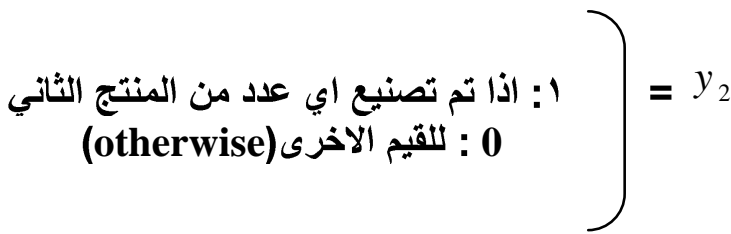

1 : اذا تم تصنيع اي عدد من المنتج الثالث

0: للقيم الاخرى(

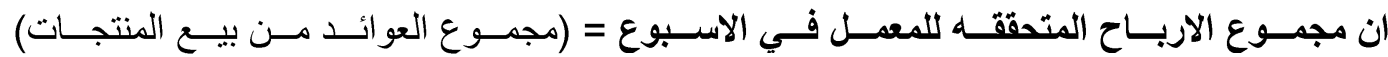

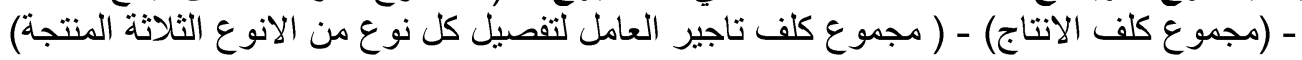

Weekly profit $=\left(12 X_{1}+8 X_{2}+15 X_{3}\right)-\left(6 X_{1}+4 X_{2}+8 X_{3}\right)-\left(200 y_{1}+150 y_{2}+100 y_{2}\right)$

$$
\text { تصبح دالة الهُف: (1- (4)...... }
$$

Maximize

S.T

$3 X_{1}+2 X_{2}+6 X_{3} \leq 150 . .($ Labor - constraint $) \ldots \ldots . .(4-2)$

$4 X_{1}+3 X_{2}+4 X_{3} \leq 160$..Cloth - constraint)...(4-3)

$X_{1}, X_{2}, X_{3} \geq 0, .$. and.int eger...........(4-4)

$y_{1}, y_{2}, y_{3}=0$..or...1..............(4-5) 


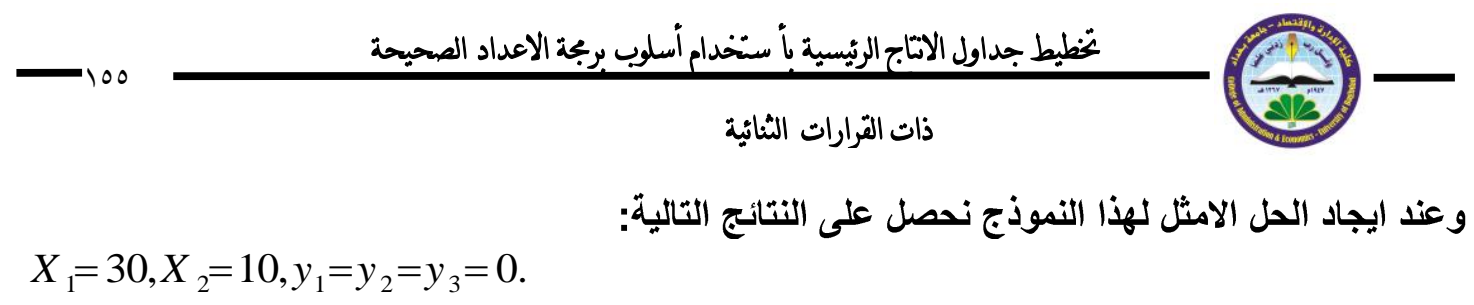

ان هذا الحل غير منظقي اي عكس القيود المفروضة لان وجود انتاج يجب ان تكون هناك قيم

للمتغيرات

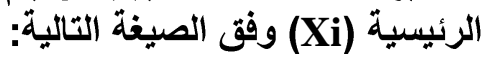
$X_{1} \leq M_{1} y_{1} \ldots \ldots \ldots \ldots \ldots . .(4-6)$
$X_{2} \leq M_{2} y_{2} \ldots \ldots \ldots \ldots \ldots . .(4-7)$
$X_{3} \leq M_{3} y_{3} \ldots \ldots \ldots \ldots \ldots . .(4-8)$

let $M_{1}, M_{2}, M_{3}$ threel arg epositiven umber

حيث تمثل (Mi) قيم كبيزة موجبة

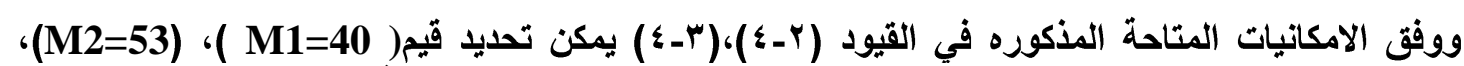

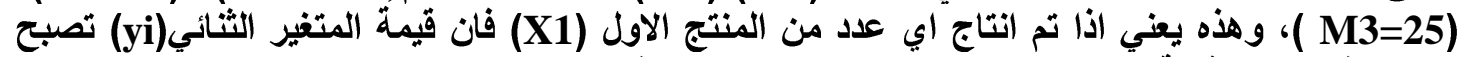

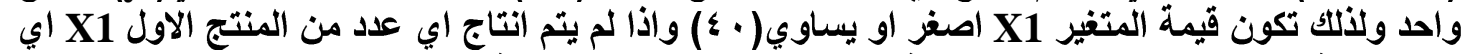

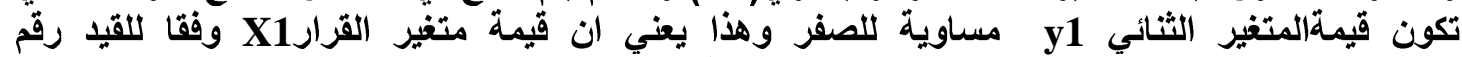

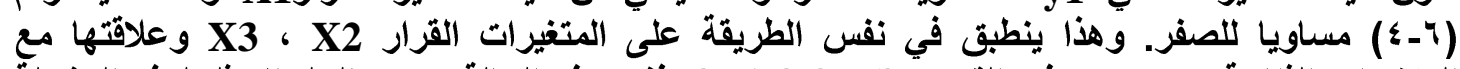

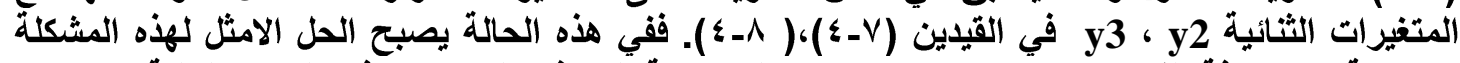
الانتاجية بعد اضافة القيود (T) $Z=\$ 75, X_{3}=25$ unites, $y_{3}=1, X_{2}=X_{3}=y_{1}=y_{2}=0$

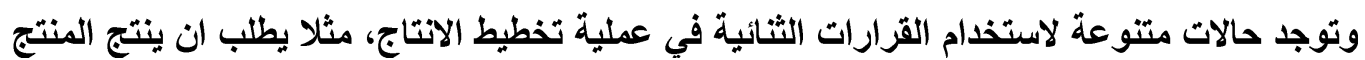

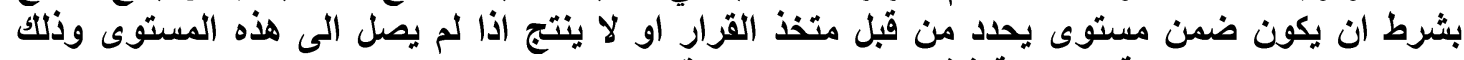

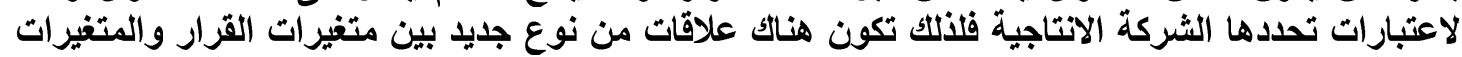

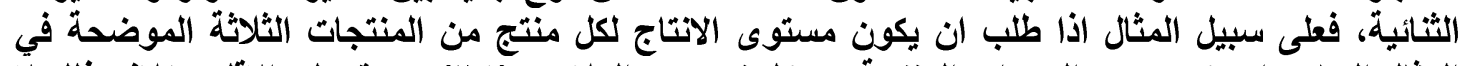

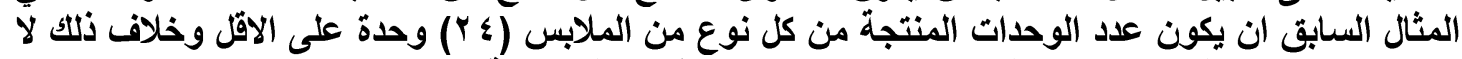

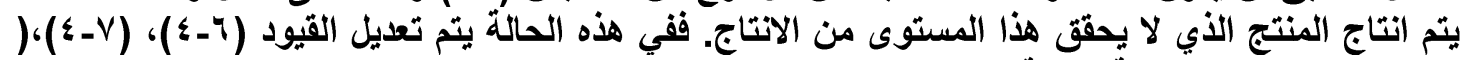

$X_{1} \leq M_{1} y$ .$(4-6)$

$24-X_{1} \leq M_{1}\left(1-y_{1}\right)$

1- ؛ ) لتصبح على الصيغة التالية:

$X_{2} \leq M_{2} y_{2_{1}}$

$24-X_{2} \leq M_{2}\left(1-y_{2}\right)$

يصبح القيد (V- \&) على الثكل الاتي:

$X_{3} \leq M_{3} y_{3}$

$24-X_{3} \leq M_{3}\left(1-y_{3}\right)$ يصبح القيد (־-ـ ) على الثكل الاتي: يصبح القيا (^-ـ ) على الثكل الاتي: 


\section{0- التخطيط الاجمالي للانتاج}

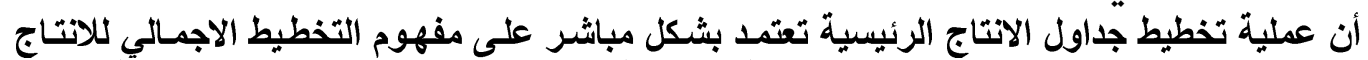

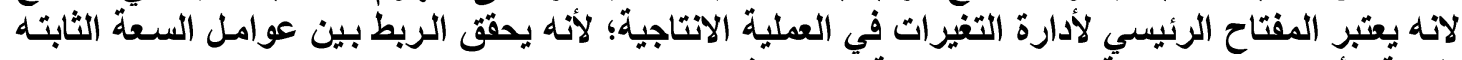

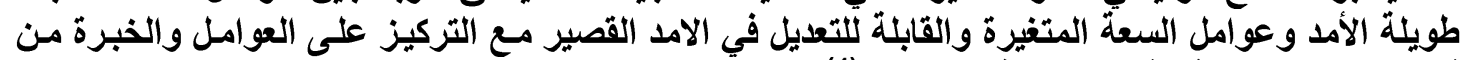

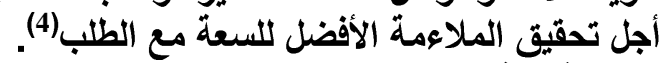

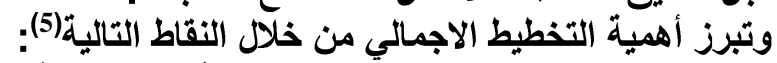

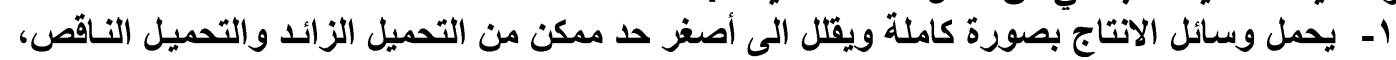

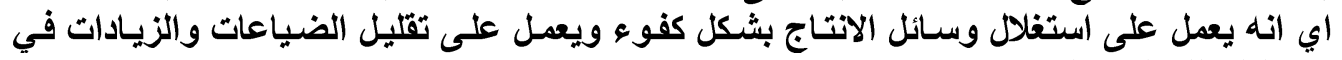
استغلال تلثك الوسيائل.

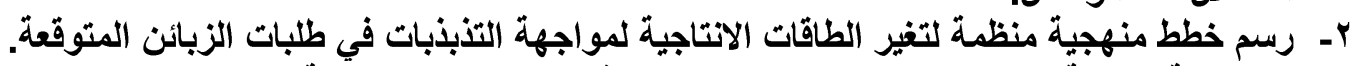

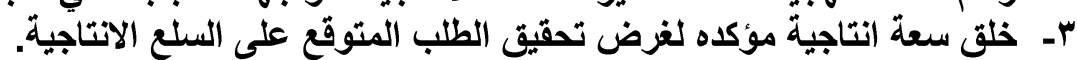

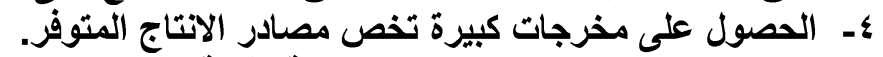

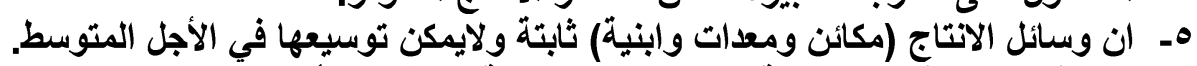

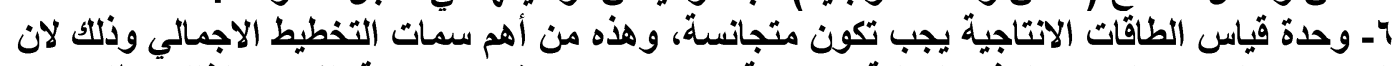

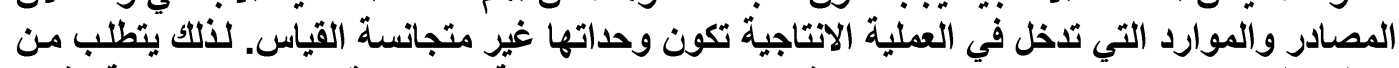

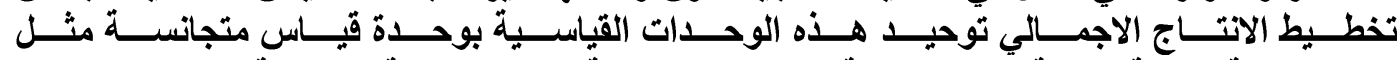

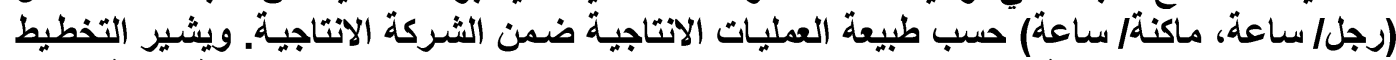

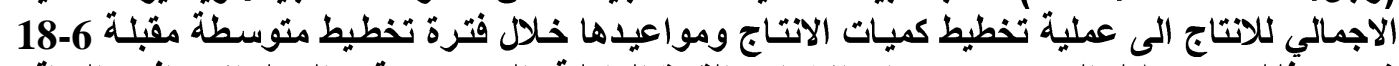

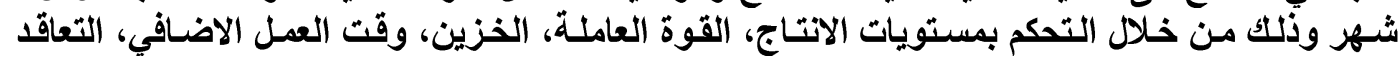
الفرعي، وبعض المتغيرات التي يمكن السيطرة عليها.

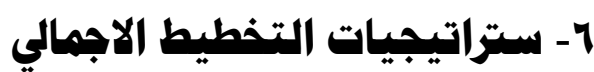

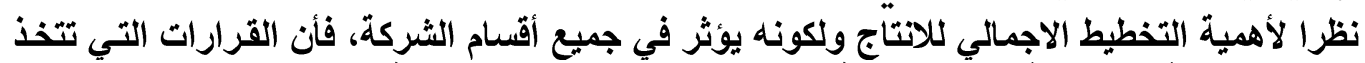

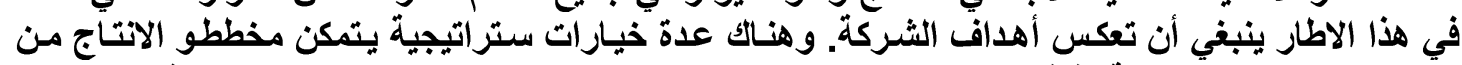

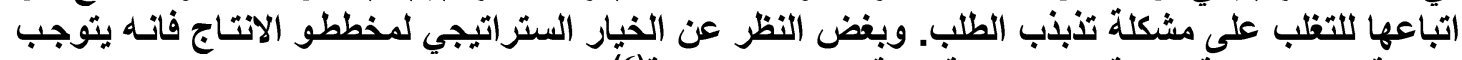

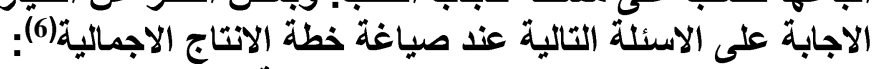

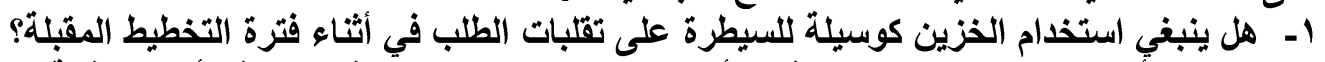

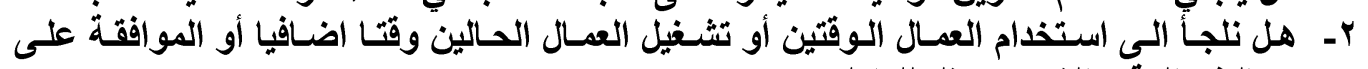

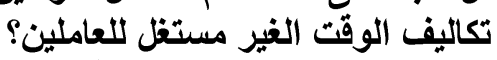

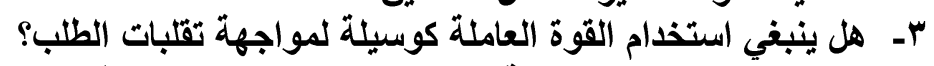

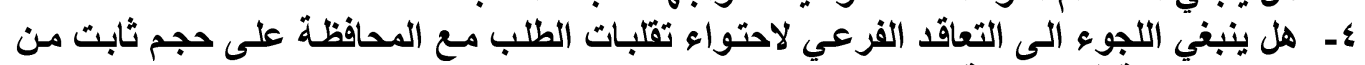

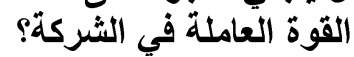
هـ هل تلجأ الى تغير الأسعار أو تغير عوامل معينة من أجل التأثير في الطلب؟

أن جميع الأسئلة السابقة تمثل ستراتيجيات مشروعة متاحة للتخطيط الاجمـالي حيث تتضمن التحكم

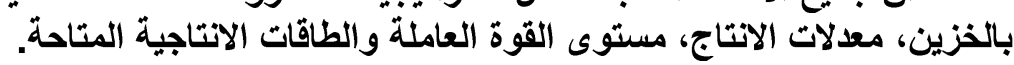




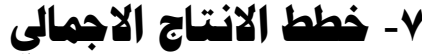

لغرض اعداد خطط الاتتاج الاجمالية للثركات الصناعية لابد من عمل نماذج رياضية تأخذ بنظر الاعتبار جميع

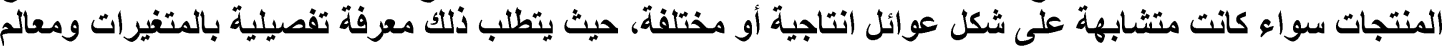

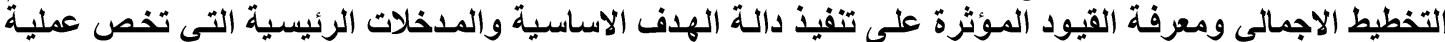

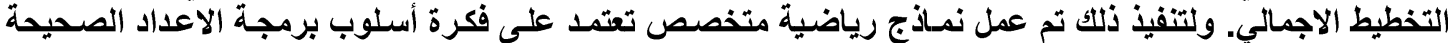

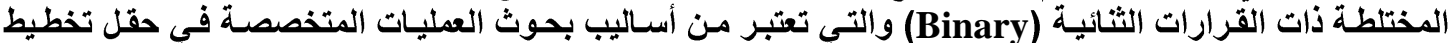

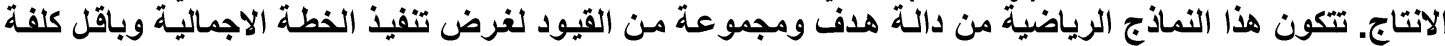

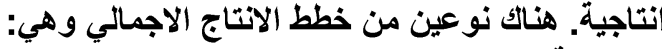

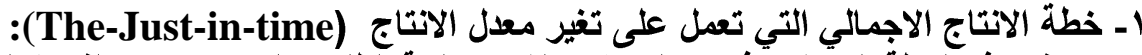

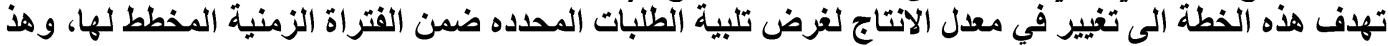

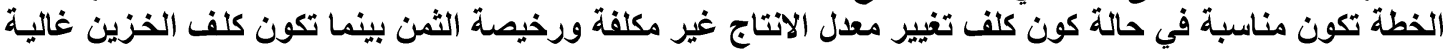

ولتوضيح ذلت ناخذ البيانات الموضحة في الجدول ادناه

\begin{tabular}{|c|c|c|c|c|c|c|c|c|}
\hline & Dec & Jan & Feb & Mar & Apr & May & Jun & Totals \\
\hline Working days (ايام العمل) & & 20 & 24 & 18 & 26 & 22 & 15 & \\
\hline Demand (الطلبات) & & 1280 & 640 & 900 & 1200 & 2000 & 1400 & \\
\hline Hiring (عدد العمال المستقدمين) & & $\mathbf{0}$ & $\cdot$ & 17. & $\cdot$ & $r .0$ & rq. & Voo \\
\hline Firing (عدد العمال المستغنى عنه) & & $r \leqslant$ & $\Lambda \leqslant$ & - & YV & - & • & $1 \leqslant 0$ \\
\hline Workforce (حجم العمل) & 300 & 266 & 182 & 342 & 315 & 620 & 910 & \\
\hline Production (الوحدات المنتجة) & & $\checkmark \wedge$. & $7 \leqslant$ & $9 \cdot r$ & $1 Y \ldots$ & 1999 & r... & \\
\hline الوحدات المخزونة (الونة) & 500 & $\mathbf{0}$ & $\mathbf{0}$ & 2 & 2 & $\mathbf{0}$ & 6.1 & 604 \\
\hline
\end{tabular}

Table1-1-: the just-in-time Production Plan

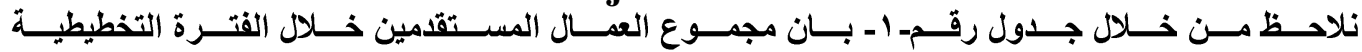

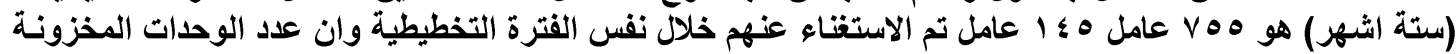

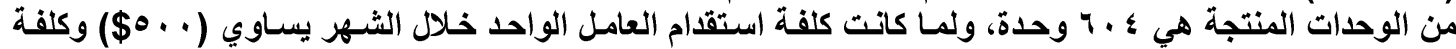

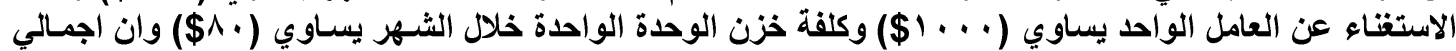

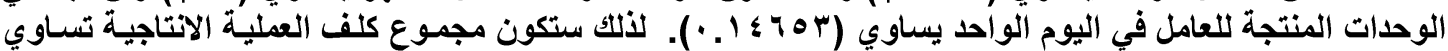

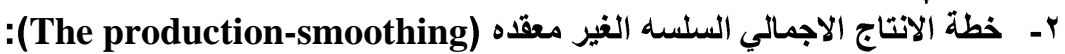

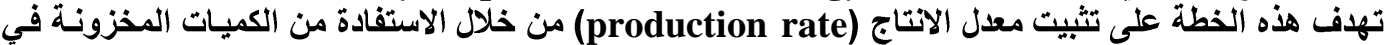

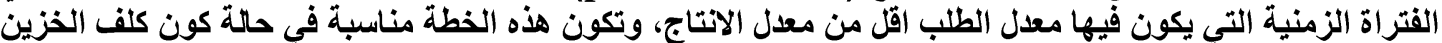

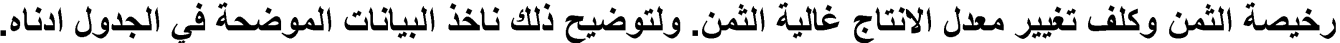

\begin{tabular}{|c|c|c|c|c|c|c|c|c|}
\hline & Dec & Jan & Feb & Mar & Apr & May & Jun & Totals \\
\hline Working days (ايام العمل) & & 20 & 24 & 18 & 26 & 22 & 15 & \\
\hline Demand (الطلبات) & & 1280 & 640 & 900 & 1200 & 2000 & 1400 & \\
\hline Hiring (عدد العمال المستقدمين) & & 111 & $\cdot$ & $\mathbf{0}$ & $\cdot$ & $\mathbf{0}$ & $\mathbf{0}$ & 111 \\
\hline Firing (عدد العمال المستغنى عنه) & & - & . & . & $\cdot$ & - & - & . \\
\hline Workforce (حجم العمل) & 300 & 266 & 182 & 342 & 315 & 620 & 910 & \\
\hline Production (الوحدات المنتجة) & & $\vee \wedge$. & T & $9 \cdot r$ & $1 \% \ldots$ & 1999 & r... & \\
\hline Inventory (الوحدات المخزونة) & 500 & $\leqslant Y \leqslant$ & Irr. & $1 \leq 1 \leq$ & $I \vee \wedge$. & 11.0 & $6 \cdot 1$ & 9071 \\
\hline
\end{tabular}

Table-2-: the Production-Smoothing Plan 


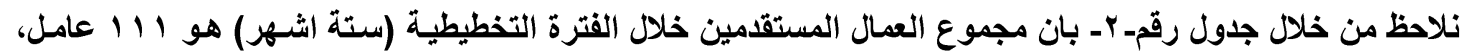

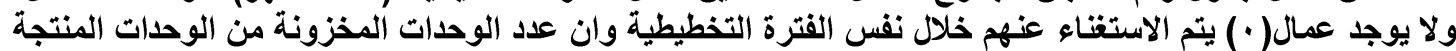

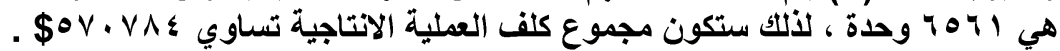

\section{A- نهاذج تخطيط الانتاج الاجمالي}

وقد تم تطوير نوعين من الموديلات الرياضية لخطط الانتاج الاجمالي عن طريق استخدام اسلوب البرمجة

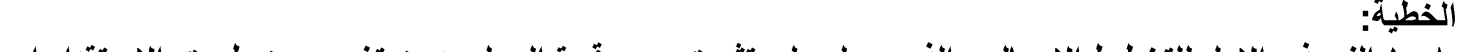
^- أ النموذج الاول للتخطيط الاجمالي: الذي يعمل على تثبيت حجم قوة العمل بدون تغيير عن طريق الاستقدام او

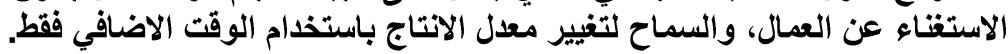

تعريف المتغيرات والثوابت الثعات والثيات

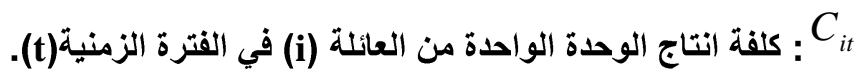

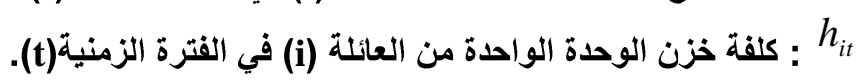

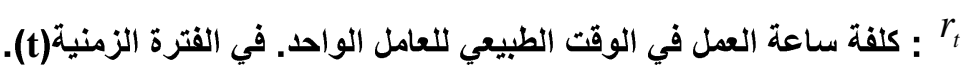
كلفة ساعة العمل في الوقت الاضافي للعامل الواحل. في الفترة الزمنية(t) : ${ }^{o}{ }_{t}$

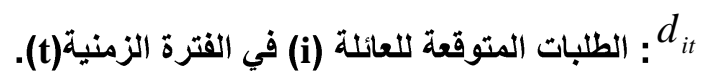

(i) : الوقت المطلوب (رجل ساعة)لاتتاج وحدة واحدة للعائلة : $M_{i}$

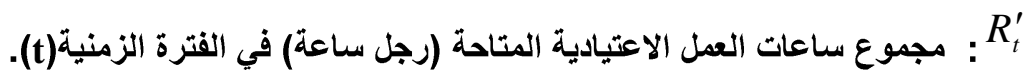
مجموع ساعات العمل الاضافية المتاحة (رجل ساعة) في الفترة الزمنية(t).

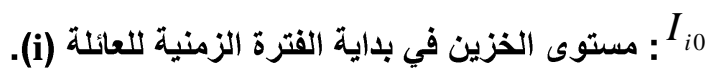
T

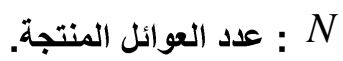
متغيزات القرار

(t) : $X_{i t}$

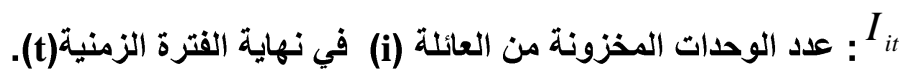

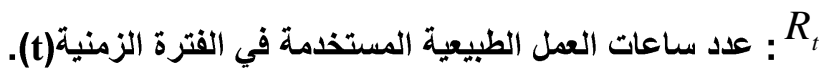

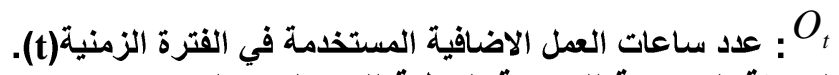
الصيغة الرياضية للبرمجة الخطية للموديل الاول:

Minimize $\sum_{i=1}^{N} \sum_{t=1}^{T}\left[c_{i t} X_{i t}+h_{i t} I_{i t}\right]+\sum_{t=1}^{T}\left[r_{t} R_{t}+o_{t} O_{t}\right]$ 
Subject to:

$$
\begin{array}{r}
X_{i t}+I_{i, t-1}-I_{i t}=d_{i t} \forall i, t \ldots . \\
\sum_{i=1}^{N} M_{i} X_{i t}-R_{t}-O_{t}=0 \forall t \\
0 \leq R_{t} \leq R_{t}^{\prime} \forall t \ldots . \\
X_{i t} \geq 0, I_{i t} \geq 0 \forall i, t .
\end{array}
$$

\begin{tabular}{|c|c|c|c|c|c|c|}
\hline & Jan & Feb & Mar & Apr & May & Jun \\
\hline $\operatorname{Demand}\left(d_{i t}\right)$ & 100 & 100 & 150 & 200 & 150 & 100 \\
\hline Unit production cost $\left(C_{i t}\right)$ & 7 & 8 & 8 & 8 & 7 & 8 \\
\hline Unit-holding $\operatorname{cost}\left(h_{i t}\right)$ & $r$ & $\varepsilon$ & $\varepsilon$ & $\varepsilon$ & $r$ & T \\
\hline Unit-regular labor cost $\left({ }^{r_{t}}\right)$ & 10 & 10 & 11 & 11 & 10 & 10 \\
\hline Unit-overtime labor $\operatorname{cost}\left({ }^{o_{t}}\right)$ & 22.5 & 22.5 & YV & YV & 22.5 & 22.5 \\
\hline Available man-hours regular $\left(R_{t}^{\prime}\right)$ & Ir. & $1 \pi$. & IT. & 10. & $1 \ldots$ & $1 \ldots$ \\
\hline Available man-hours overtime $\left({ }^{\prime}{ }_{t}^{\prime}\right)$ & $r$. & $\xi$. & $\varepsilon$. & $r$ & $r$. & $r$. \\
\hline
\end{tabular}

\begin{tabular}{|c|c|c|c|c|c|c|c|}
\hline & Dec & Jan & Feb & Mar & Apr & May & Jun \\
\hline Man-hours (R) labor & & 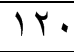 & $1 T$ & $1 \%$. & 10. & $1 \ldots$ & $1 \ldots$ \\
\hline Man-hour( O )labor & & • & IV & . & $r$. & $r$. & . \\
\hline Production $\left(X_{i t}\right)$ & & Ir. & $1 \leqslant V$ & Ir. & 11. & $1 \pi$ & $1 \ldots$ \\
\hline Inventory $\left(I_{i t}\right)$ & $r$ & rT & $V$. & $\varepsilon$. & $r$. & . & . \\
\hline Optimal cost & \multicolumn{7}{|c|}{$\$(20193)$} \\
\hline
\end{tabular}

\section{Table - r- Data for Model 1}

ومن خلال استخدام المعادلات الرياضية للموديل الاول نحصل على الحل الامثل الموضح في الجدول رقم (ع).

Table - \& - Solution to Model 1 


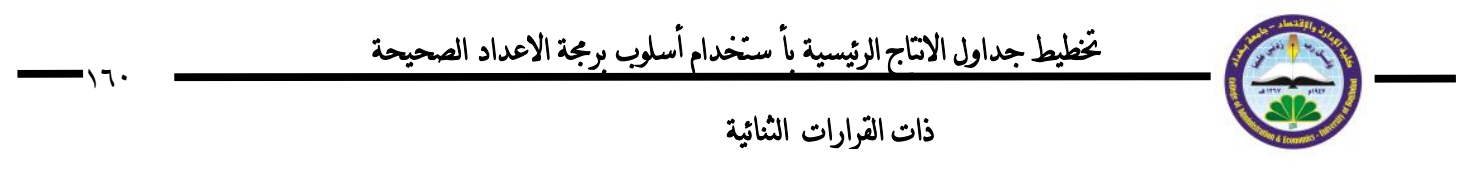

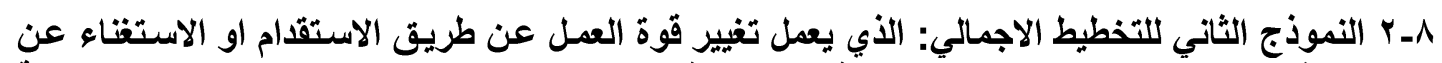

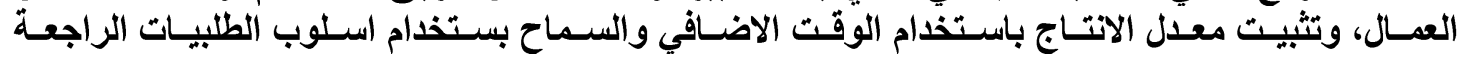
.(Backorder)

تعريف المتغيرات والثوابت

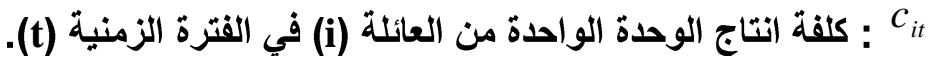

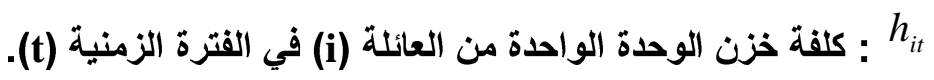

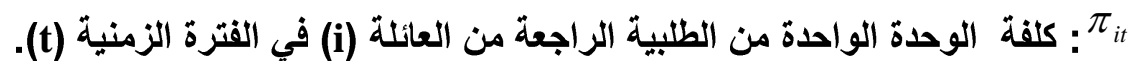

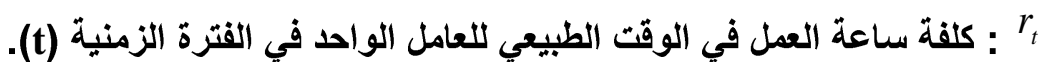
(t) : كلفة ساعة العمل في الوقت الاضافي للعامل الواحد في الفترة الزمنية : ${ }^{o}{ }_{t}$

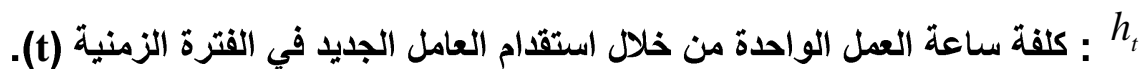

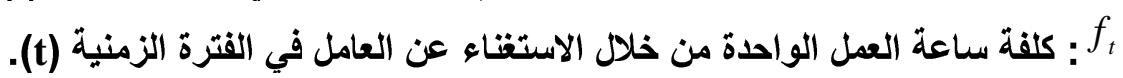
الطلبات المتوقعة للعائلة (it $d_{i t}$

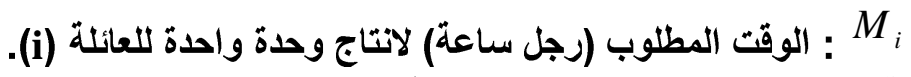

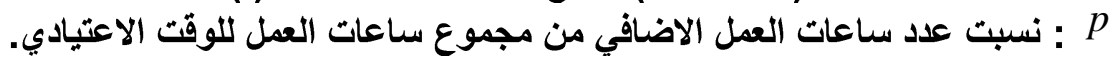

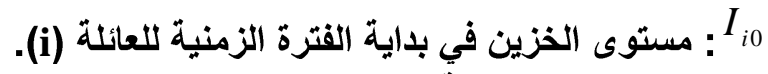

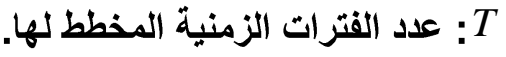
N متغيرات القرار

(it $X_{i t}$ : : $R_{t}$

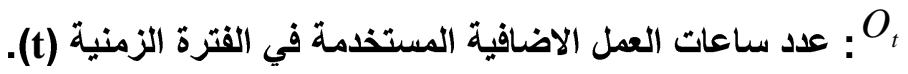

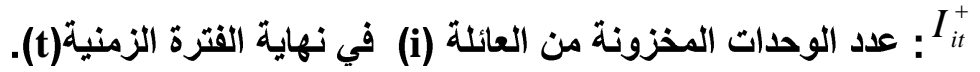

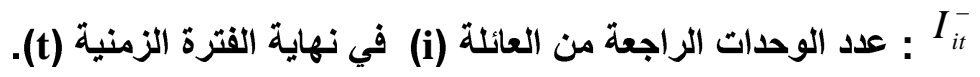
F ${ }_{t}$ : : عداعد : : $F_{t}$ 


\section{دالةُّ الهيفة الرياضية للبرمجة الخطية للموديل الثاني}

Minimize $\sum_{i=1}^{N} \sum_{t=1}^{T}\left[c_{i t} X_{i t}+h_{i t} I_{i t}^{+}+\pi_{i t} I_{i t}^{-}\right]+\sum_{t=1}^{T}\left[r_{t} R_{t}+o_{t} O_{t}+h_{t} H_{t}+f_{t} F_{t}\right]$

\section{Subject to:}

$X_{i t}+I_{i, t-1}^{+}-I_{i t}^{+}-I_{i, t-1}^{-}+I_{i t}^{-}=d_{i t} \forall i, t$

$\sum_{i=1}^{N} M_{i} X_{i t}-R_{t}-O_{t} \leq 0 \forall t$

$R_{t}-R_{t-1}-H_{t}+F_{t}=0 \forall t$

$O_{t}-p R_{t} \leq 0 \forall t$

$X_{i t} \geq 0, . . I_{i t}^{+} \geq 0, . . I_{i t}^{-} \geq 0 \forall i, t$

$R_{t, . .} O_{t}, . . H_{t}, . . F_{t} \geq 0 \forall t$.

\begin{tabular}{|c|c|c|c|c|c|c|}
\hline & Jan & Feb & Mar & Apr & May & Jun \\
\hline $\operatorname{Demand}\left(d_{i t}\right)$ & 100 & 100 & 150 & 200 & 150 & 100 \\
\hline Unit production cost $\left(C_{i t}\right)$ & 7 & 8 & 8 & 8 & 7 & 8 \\
\hline Unit-holding $\operatorname{cost}\left(h_{i t}\right)$ & $r$ & $\xi$ & $\varepsilon$ & $\varepsilon$ & $r$ & $r$ \\
\hline Unit-regular labor $\operatorname{cost}\left({ }^{r_{t}}\right)$ & 10 & 10 & 11 & 11 & 10 & 10 \\
\hline Unit-overtime labor $\operatorname{cost}\left({ }^{o_{t}}\right)$ & 22.5 & 22.5 & TV & TV & 22.5 & 22.5 \\
\hline Unit Backorder cost $\left(\pi_{i t}\right)$ & 20 & 25 & 25 & 25 & $r$. & 10 \\
\hline Hiring $\operatorname{cost}\left(h_{t}\right)$ & 20 & $r \cdot$ & r. & $r \cdot$ & $r$ r. & $r \cdot$ \\
\hline Firing $\operatorname{cost}\left(f_{t}\right)$ & $r \cdot$ & $r \cdot$ & $r \cdot$ & $r \cdot$ & $r$. & $r \cdot$ \\
\hline
\end{tabular}

Table - ๑- Data for Model 2 
من خلال استخدام المعادلات الرياضية للموديل الثاني للتخطيط الاجمالي نحصل على الحل الامثل الموضح في

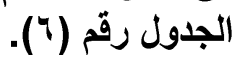

\begin{tabular}{|c|c|c|c|c|c|c|c|}
\hline & Dec & Jan & Feb & Mar & Apr & May & Jun \\
\hline Man-hours Hired( $\left.H_{t}\right)$ & & 129 & 0 & 0 & 0 & 0 & 0 \\
\hline Man-hour Fired $\left(F_{t}\right)$ & & . & 0 & . & 0 & 0 & V \\
\hline Man-hours (R) labor & & 1199 & 149 & 119 & 1149 & 1199 & $|M|$ \\
\hline Man-hour( O )labor & & - & $\cdot$ & $\cdot$ & rt & . & $\cdot$ \\
\hline Production $\left(X_{i t}\right)$ & & 119 & 119 & 1199 & 171 & 119 & $|M|$ \\
\hline Inventory $\left(I_{i t}^{+}\right)$ & $r$ & r & 7. & $r q$ & . & . & $\cdot$ \\
\hline Units of Backorder( $\left.I_{i t}^{-}\right)$ & & . & . & . & . & YI & $\cdot$ \\
\hline Optimal cost & \multicolumn{7}{|c|}{$\$(19784)$} \\
\hline
\end{tabular}

Table - - Solution to Model 2

9- نماذج تبرزئة التضطيط الاجمالي (Disaggregate models):

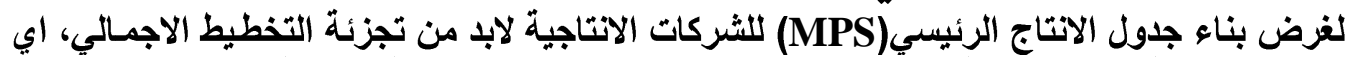

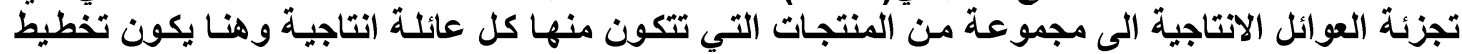

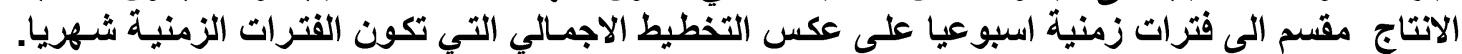

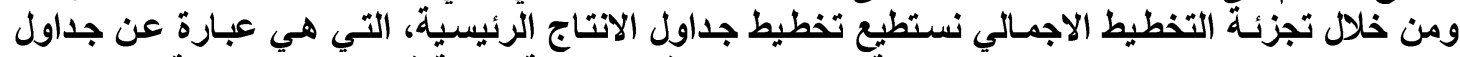

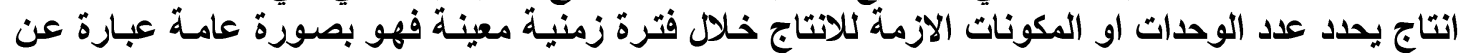

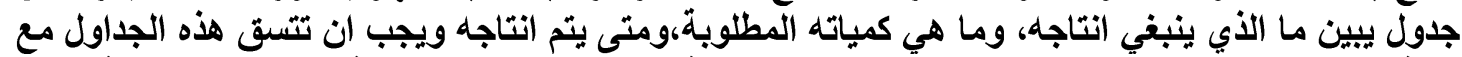

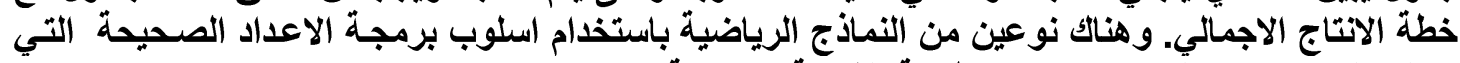

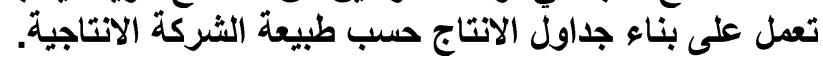

9ـ ا نموذج جداول الانتاج لغرض الخزين( Optimizing MPS in a make-to-stock model)

\section{تعريف المتغيرات والثوابت:}

n: thenumberofparts ( عدد المنتجات التي تنكون منها كل عائلة المطلوب انتاجه )

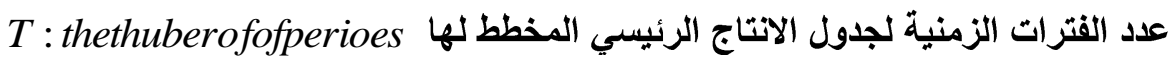

$A_{i}:$ set - up cos tforproducti (كلقة تتصيب المنتجات)

$h_{i}$ : holding cos tperunit (كلقة خزن الوحدة الواحدة من المنتجات خلال كل فترة زمنية)

$a_{i}$ : productionhouresrequiredperunitofproducti

الساعات المطلوبة للانتاج وحدة واحدة من منتجات كل عائلة من العوائل الاتتاجية

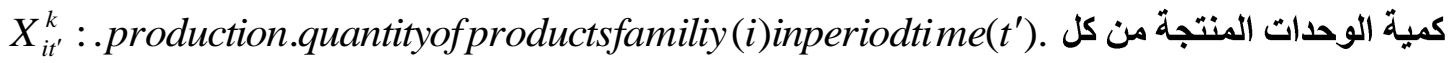

منتج من منتجات العوائل الانتاجية خلال الفترات الزمنية المخطط لها( وتكون طول الفترة اسبوع) 
$I_{i t}$ :onhandinventoryofproductiatthe endofperiodt (كمية الخزين من كل منتج في نهاية الفترة ) $Y_{i t}=1$ ifproducti isproducedinperiodt,0otherwise ويساوي صفر في حالة عدم وجود انتاج في كل فترة زمنية

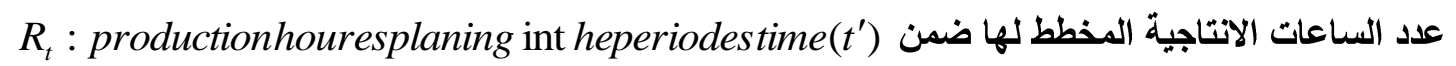
الثهز الواحد والتي ثم استنتاجها من نماذج التخطيط التطن الاجمالي

\section{الصيغة الرياضية للموديل الاول:}

$$
\begin{aligned}
& \text { MinZ }=\sum_{i=1}^{n} \sum_{k=1}^{K} \sum_{t^{\prime}=1}^{T}\left(A_{i}^{k} Y_{i t^{\prime}}+h_{i}^{k} I_{i t^{\prime}}^{k}\right): \text { دالة الهزف } \\
& I_{i t^{\prime}}^{k}=X_{i t^{\prime}}^{k}+I_{i t^{\prime}-1}^{k}-d_{i t^{\prime}}^{k}: \text { ق. قيد الخزين } i=1 . . . n, . . t^{-}=1, \ldots, t \quad k=1, \ldots K \\
& \sum_{i=1}^{n} \sum_{k=1}^{K} a_{i}^{k} X_{i t^{\prime}}^{k} \leq 1 / 4\left(R_{t}\right) \text { قيل الطاقات الانتاجية } \quad \mathbf{t}=\mathbf{1}, \mathbf{T}, i=1 . . n, . t^{-}=1, \ldots, t k=1, . K \\
& X_{i t^{\prime}}^{k} \leq M Y_{i t^{\prime}}^{k} \text { قيد مستوى الانتاج } \\
& k=1, \ldots K i=1 \ldots . . n, . . t^{-}=1
\end{aligned}
$$

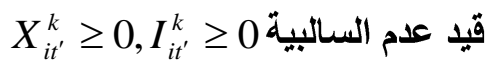

$$
\begin{aligned}
& k=1, \ldots K i=1 \ldots . . . . . t^{-}=1 \text {, } \\
& Y_{i t^{\prime}}^{k}=(0,1) \\
& k=1, \ldots K i=1 \ldots . . . . . t^{-}=1, \ldots, t \text {. }
\end{aligned}
$$

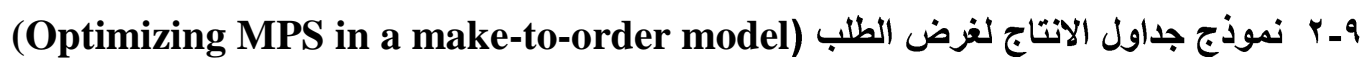

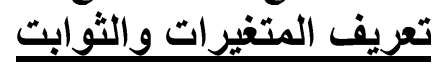
فترة زمنية من الوحنت المنتجة من كل منتج من منتجات العبوات العوائل الانتاجية المطلوب انتاجها في كل $X_{i t}^{k}$

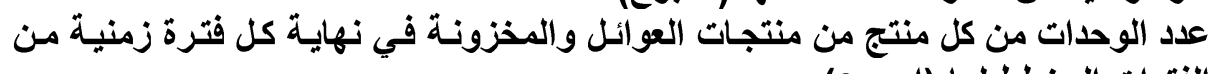
الفترات المخطط لها (اسبوع) $I_{i t^{\prime}}^{k}$ عدد الوحدات من كل منتج من منتجات المطات العوائل الاتتاجية الغير متحققه في كل فترة زمنيـة

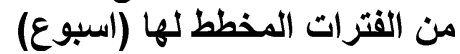
وقت العمل الغير مستثل في كل فترة زمنية من الفترات الفخرة المخطط لها (اسبوع) $I_{i t^{\prime}}^{-k}$ $d_{t^{\prime}}^{0-}$ C $\quad d_{t^{\prime}}^{0^{\prime+}}$

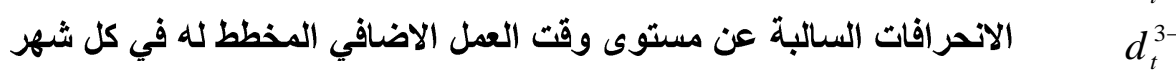

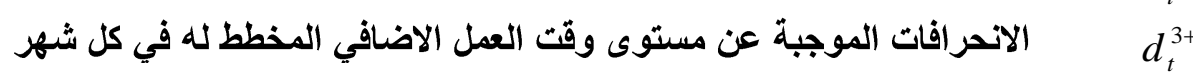

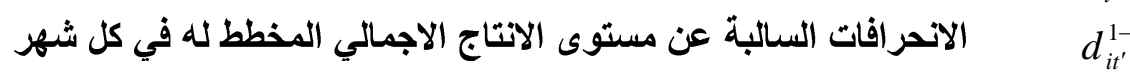

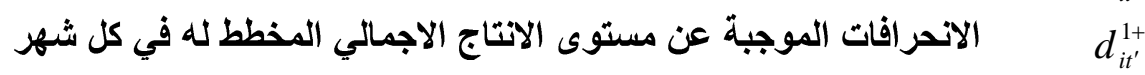

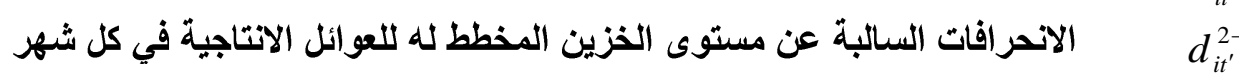

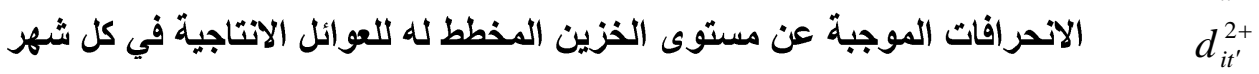


كلفة الطبية الغير متحققه للوحدة الانتاجية الغير متحققة.

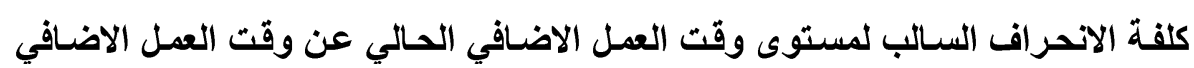

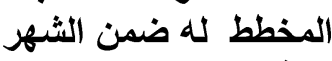

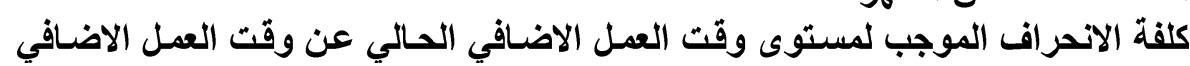

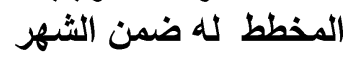

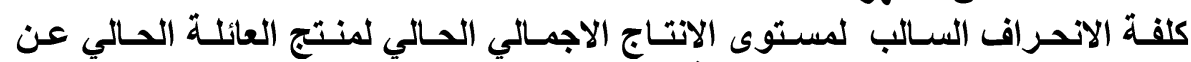

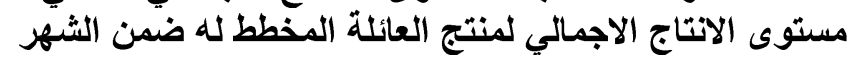

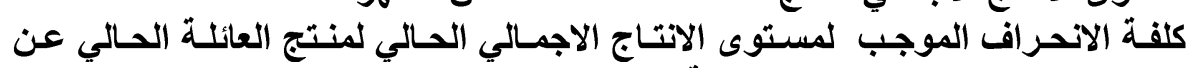

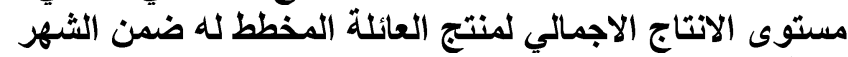

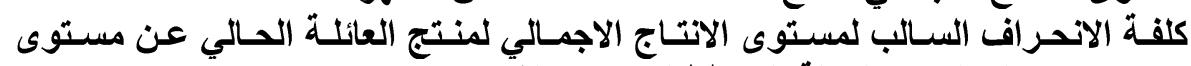

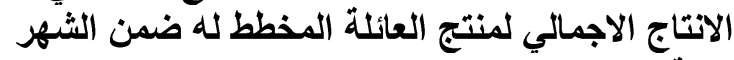

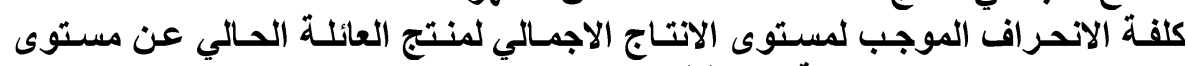

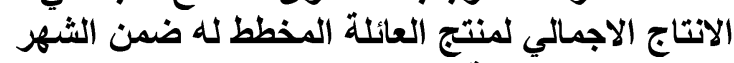
عدد الفترات الزمنية المخطط لهائج العانلة (اسبوع) عدد العوائل الانتاجية عدد المراكز الانتاجية

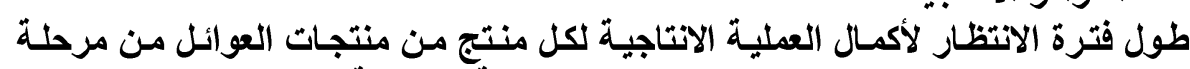

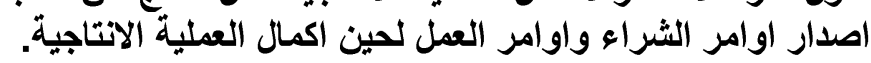

$$
\pi_{i t^{\prime}}^{k}
$$
$W^{3-}$ $W^{3+}$

$W^{1-}$

$W^{1+}$

$W^{2-}$

$W^{2+}$ $t^{\prime}$ $\mathrm{n}$ $\mathrm{J}$ $\mathrm{L}_{\mathrm{i}}$ $\mathrm{m}$

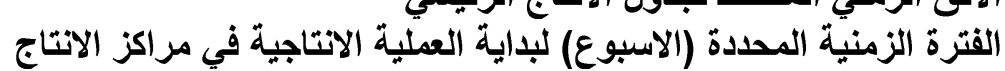

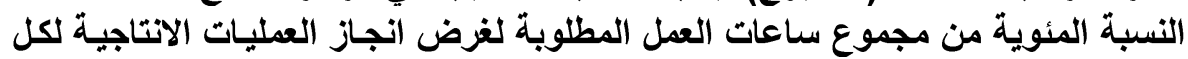
منتج من منتجات العوائل في كل مركز من مراكز العن الانتاج .

الصيغة الرياضية للموديل الثانيى

Objective function:

Minimize:

(دالة الهدف)

$\mathbf{Z}=$

$\sum_{i=1}^{n} \sum_{k \in K_{i}} \sum_{t^{\prime} \in N_{t}} \pi_{i t^{\prime}}^{k} I_{i t^{\prime \prime}}^{-k}+\sum_{i=1}^{m}\left(w^{3-} d^{3-}+w^{3+} d^{3+}\right)+\sum_{i=1}^{n} \sum_{t=1}^{m}\left(w_{i}^{1-} d_{i t}^{1-}+w_{i}^{1+} d_{i t}^{1+}+w_{i}^{2+} d_{i t}^{2+}\right)$

Subject to:

(The Inventory Constraint)

$$
\begin{aligned}
& I_{i . t^{\prime}-1}^{k}-I_{i t^{\prime}}^{k}+X_{i t^{\prime}}^{k}+I_{i t^{\prime}}^{-k}-I_{i . t^{\prime}-1}^{-k}=d_{i t^{\prime}}^{k} \ldots \ldots . . . \\
& \quad\left(\text { for } i=1, \ldots, n ; k \in K_{i} ; t^{\prime} \in N_{t} \text { and } t=1, \ldots, m\right)
\end{aligned}
$$

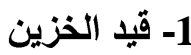


2- قيا وقت العمل الاعتيادي والاضافي (The Time Constraints)

$$
\begin{gathered}
\sum_{i}^{n} \sum_{k \in K_{i}} \sum_{m^{\prime}}^{L i} \sum_{j=1}^{J}\left(r_{i m^{\prime} j} X_{i . t^{\prime}+L i-m^{\prime}}\right)+d_{t^{\prime}}^{0-}-d_{t^{\prime}}^{0+}=(1 / 4)\left[R_{t}-\sum_{i=1}^{n} \beta z\left(X_{i t}\right] .\right. \\
\left(\text { fort }{ }^{\prime} \in N_{t} \text { andt }=1, \ldots, m\right)
\end{gathered}
$$

rـ قيد انحراف وقت العمل الاضافي (Overtime Deviation Constrain)

$\sum_{t^{\prime} \in N_{t}} d_{t^{\prime}}^{\mathrm{O}+}+d_{t}^{3-}-d_{t}^{3+}=O_{t}$

ع - قيد انحراف وقت العمل الاعتيادي (Regular Time Deviation Constraint)

$\sum_{k \in K_{i}} \sum_{t^{\prime} \in N_{t}} X_{i t^{\prime}}^{k}+d_{i t}^{1-}-d_{i t}^{1+}=X$

$($ fori $=1, \ldots$, nand $t=1, \ldots, m)$

هـ قيد انحراف الخزين(Inventory Deviation Constraint)

$\sum_{k \in K_{i}} \sum_{t^{\prime} \in N_{t}} I_{i t^{\prime}}^{k}+d_{i t}^{2-}-d_{i t}^{2+}=X_{i t}$

$$
\left(\text { for } i=1, \ldots, n ; t=1, \ldots, m ; \text { and }^{\prime}=4(t-1)+1, \ldots, 4(t-1)+4\right.
$$

צ- قيد عدم السالبية (Non-Negativity Constraint)

$X_{i t^{\prime}}^{k}, I_{i t^{\prime}}^{k}, I_{i t^{\prime}}^{-k}, d_{t^{\prime}}^{0-}, d_{t^{\prime}}^{0+}, d_{i t}^{2-}, d_{i t}^{2+}, d_{i t}^{3-}, d_{i t}^{3+}, d_{t}^{1-}, d_{t}^{1+} \geq 0$. 
اـ التخطيط الاجمالي يعتبر المفتاح الرئيسي للعمليات الانتاجية لانه يحقق الربط بين التخطيط طويل الامــ

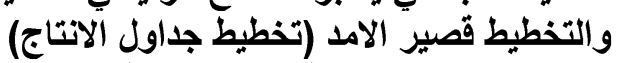

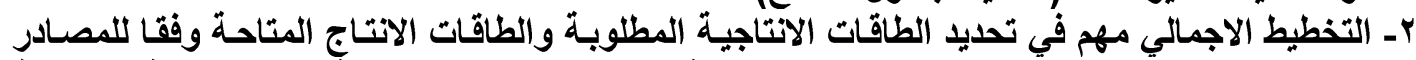

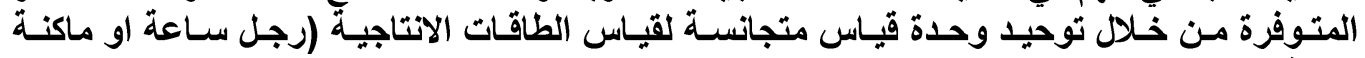
ساعة).

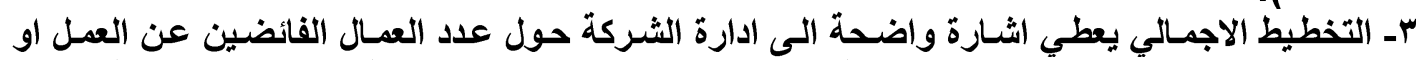

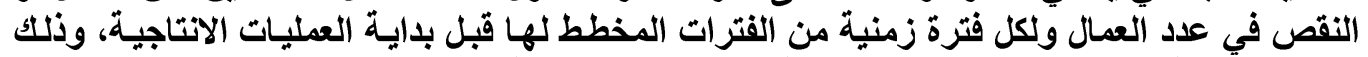

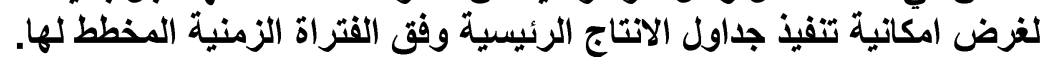

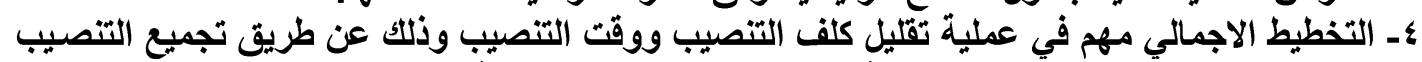

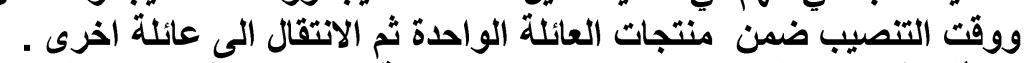

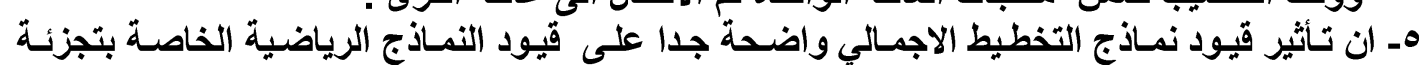

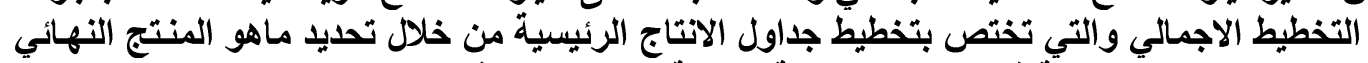

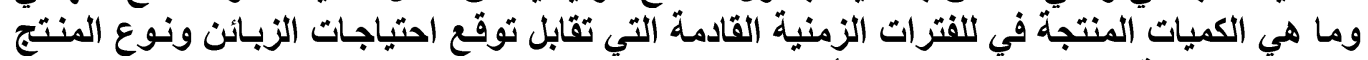

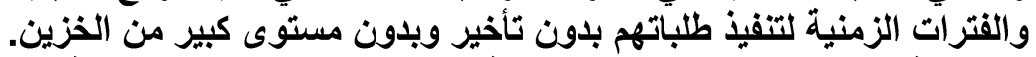

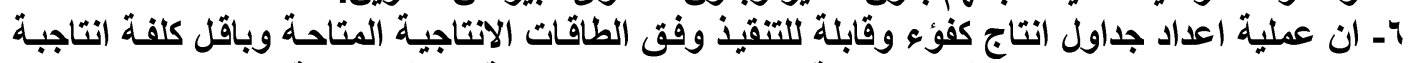

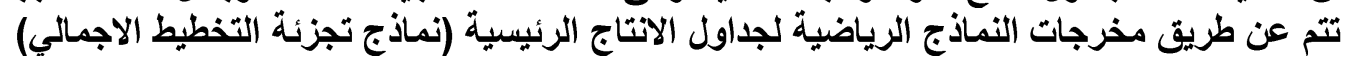

\section{|}

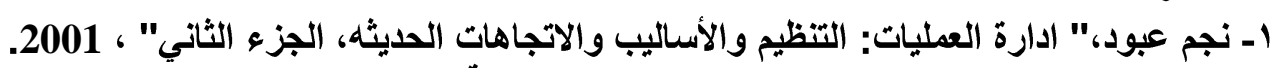

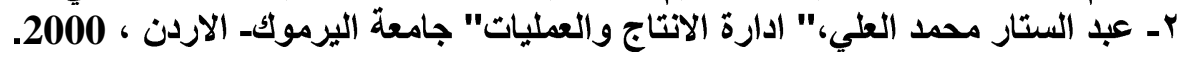
3- WAYNE L. WINSTON." operations research applications and algorithms", Indiana University, 1993.

4- Joseph G. Monks,"operations management theory and problems" 2001.

5- Zaner, J.A., "Production and Inventory Control", Department of Technology, University of Southern Mine,2003.

6- Heizer, J., and Render, B., " Operations Management" Prentice Hall, 2002.

7- James R. Evans "Applied production and operation management" 2003

8- Hillier and Lieberman "Introduction to Operations Research", Stanford University 1987.Holden-day,Inc san Francisco. 